\title{
The Scientific Study of Parents and Caregivers of Children with ASD: A Flourishing Field but Still Work to be Done
}

\author{
Anders Nordahl-Hansen ${ }^{1} \oplus \cdot$ Logan Hart ${ }^{2} \cdot$ Roald A. Øien ${ }^{2,3}$
}

Published online: 3 March 2018

(c) Springer Science+Business Media, LLC, part of Springer Nature 2018

\begin{abstract}
There is a long history of research on parents and caregivers of individuals within autism. Parents and other primary caregivers typically play the most important part in the lives of persons with ASD although the need for support as the child becomes of age varies widely. This special issue includes 30 articles on central areas related to parenting and caregiving for people with ASD. Some of the key themes include intervention and training, mental health issues related to parent and family stress, measurement and assessment, and parent-child transactional processes. Other articles in this issue consider different but equally important topics such as sibling as potential future caregivers and parent support of preschool peer relationships.
\end{abstract}

Keywords Parents $\cdot$ Caregivers $\cdot$ Parent-child interaction $\cdot$ Autism

This special issue in the Journal of Autism Spectrum Disorders focuses on parents, primary caregivers of individuals with autism spectrum disorders. We were pleased to receive over a hundred manuscripts. The high number of submissions was not unexpected given the breadth of the topic that has occupied a central position in the early years of autism research and continues to do so. Most submissions for this special issue, in one way or another focus on parents and/ or parent-child related topics within areas such as mental health and stress, intervention, parent-child interaction, and measurement. However, other parenting- and caregiverrelated topics are also addressed. All focus on topics that

Anders Nordahl-Hansen and Roald A. Øien contributed equally to the main content of the manuscript.

Anders Nordahl-Hansen

a.j.hansen@isp.uio.no

Roald A. Øien

roald.a.oien@uit.no

1 Department of Special Needs Education, University of Oslo, P.O. Box 1140, Blindern, 0318 Oslo, Norway

2 Child Study Center, Yale University School of Medicine, 230 South Frontage Road, P.O. Box 207900, New Haven, CT, USA

3 Department of Psychology, UiT - The Arctic University of Norway, 9037 Tromsö, Norway are all in need of more attention from the autism research community.

Parents go through pregnancy with increasing hope and dreams for their future child. Friends, vacations, school, education, falling in love, marriage, grandchildren, are examples of thoughts that might run through a becoming parents mind. As a parent, you cannot be prepared for the process that leads to your child receiving an ASD diagnosis. The way parents deal with this process differs widely but there are also commonalities in terms of what parents are concerned for after their child receives an ASD diagnosis. Common issues include the awareness that your child might not experience what you as a parent had hoped and dreamed for, or concerns for the future if something happens to the parents. Feelings of joy and pride in the child's accomplishments can be the same as what other parents feel although the accomplishments per se may be different. Some parents of course see their child with ASD go through the school system and higher education going on to have good secure jobs, a family of their own and friends. Nevertheless, even here the road is typically a rockier one. For instance, bullying of autistic persons in school and the workplace is common (Woodruff et al. 2017), which can lead to, or further compound mental health problems like anxiety and depression. A common challenge for primary caregivers of children with autism is seeking out the best intervention opportunities. One study in this special issue offers a glimpse into parents understanding of this less than straightforward task of choosing 
an intervention for one's child (Shepherd et al. 2017). The results of the study indicate that professionals, such as paediatricians, play an important role in the process of guiding and influencing parents' intervention choices. The authors also note that a key factor for parents when selecting specific interventions relates to the individual child's needs. Thus, there is an important responsibility resting on professionals to provide to the parents updated information on best-practice interventions that address these individual needs. This is of course not an easy task as there is a lack of evidence supporting one-size-fits-all type intervention approaches and the access and quality of services may vary widely based on location. Also, professionals may need to aid parents in finding specific interventions to deal with specific problems. The importance of interventions targeting mastery of confined skills such as toilet-training cannot be overstated. Another frequent challenge relates to sleep difficulties; these impact a substantial proportion of children with ASD, and, of course, their parents as well. One study in this issue compared two parent-implemented intervention approaches intended to enhance sleep in children with ASD where the bedtime fading approach were reported to increase children's sleep duration as well as decreasing sleep onset latency (Delemere and Dounavi 2017). The difference a good night's sleep makes should not be under-estimated.

Issues of measurement and definition are crucial to the scientific endeavour. Various types of psychometric quality indicators such as validity and reliability are of course essential for a measure's utility. Another question is to what extent a measure has the sensitivity and/or specificity to detect what it is set out to detect, whether it be false-positives or false negatives for screening-instruments, or the sensitivity to effects of change following treatment. When it comes to instruments measuring change in for instance social communication intervention studies, the quantity of tools available is not the problem (there are too many, making comparison across studies difficult) but rather the quality and low consensus of what these tools should measure (Bolte and Diehl 2013; Cunningham 2012). For many researchers, an ideal scenario following early intervention for children with autism is typically to want to find a decrease in core ASD impairments in the hope that such changes cascade onto other areas of functioning. Thus, it is natural to make use of tools that measure these core areas of impairment. However, at the end of the day (literally), it is not the interventionist but the child, parents and families that will experience the effects possible effects following interventions. In this respect an important inquiry is: What child outcomes are valued by parents of children with ASD? This is the question raised by McConachie and colleagues in this issue. The results from this study indicate that parents value a higher focus on measuring outcomes related to daily living skills rather than symptom reduction in characteristic
ASD impairments (McConachie et al. 2017). The study includes a top 10 rank of constructs parents view as important measurement of progress or outcome, happiness being ranked at number 1. A good example of a user-generated measure is presented in one of the two articles from the PACT-consortium in this special issue (Leadbitter et al. 2017). The present issue also includes two separate studies that compare parental scores to other sources of information on early ASD behaviours and symptoms (Larsen et al. 2017; Macari et al. 2017). The results from these studies are in line with other recent investigations of parent-reports that demonstrate the importance of parents and caregivers as invaluable sources of information on their child's development (Miller et al. 2017; Sacrey et al. 2018).

Several articles in the current issue address mental health in relations to caregiving roles. The studies have relatively large sample sizes and investigate interesting and important areas such as potentially protective factors against distress for caregivers (Lindsey and Barry 2018) and association between resilience and well-being of mothers of ASD and other developmental disabilities (Halstead et al. 2018). Another study addresses Parental challenges in supporting peer-relationships for their children with ASD showing that there are parent resilience factors but high demands in providing good peer opportunities for their preschool children (Estes et al. 2017).

The study by Nuttall et al. (2018) is a good example of how caregiving extends to more than parents. Their study explores how typically developing adult siblings of persons with ASD view their possible future roles as primary caregivers, an area that needs more focus of attention in the future. Another paper addresses functioning in siblings of youth with ASD. An issue one of the guest editors for this special issue can personally relate to with a daughter with autism currently entering adolescence and her three younger siblings. In this study Tudor et al. (2017) present a model that includes ASD-, Family- and sibling-variables and lay out some future directions for research and clinical practice. As many other papers in this special issue, the Tudor et al. study highlight how multiple factors interconnect and need to be taken into account to get a deeper understanding of developmental transaction processes and family dynamics.

\section{Some Issues for the Future}

Decades of work from researchers, clinicians, practitioners, and knowledge and insights from parents, caregivers and autistic people keep moving the field forward in shaping current theory, research and practice. Focus on parents and caregivers is no longer a neglected research area but some areas could benefit from more explorations in the future. More research on parents and caregivers in the typical non-western 
societies would be very welcome. Further, understanding cultural differences becomes no less important in a world with increasing mobility and migration (Fox et al. 2017), and cultural factors can be crucial to take into account when it comes to effectively implementing parent-educative courses or interventions of any sort. Additionally, culture differences can impact results following assessments and clinical decision making. It is also important that assessment tools are culture sensitive. Most studies with parent samples solely consist of mothers or an overwhelmingly large number of mothers compared to fathers. More studies are needed that focus on fathers of children with ASD. For instance, it would be interesting to explore in further depths fathers play strategies using similar observational techniques as deployed by Shire et al. (2017) in this issue. More investigations on females with ASD are also needed. A paper in this issue presents parental perspectives on what challenges their daughters with ASD may face (Mademtzi et al. 2017). Since the reasons for the sex-ratio between females and males with ASD are not fully understood it is important that researchers, in addition to investigating biological and genetic aspects, also continue to shed light on sex-differences in regards to behavioural phenotypes.

We thank all the authors that sent their respective manuscripts to this special issue. We are indebted to the reviewers that contributed with their thoughtful considerations on the various manuscripts. Special thanks go to the editorial and production staff at JADD and Springer for excellent support and cooperation. We dedicate this special issue to the parents, caregivers and their children with ASD.

Author Contributions ANH and RAØ have contributed equally in the process of writing the manuscript. LH has contributed with ideas, design, writing and comments to the manuscript.

\section{Compliance with Ethical Standards}

Conflict of interest None of the authors report having any conflicts of interest.

Ethical Approval This article does not contain any studies with human participants or animals performed by any of the authors.

\section{References}

Bolte, E. E., \& Diehl, J. J. (2013). Measurement tools and target symptoms/skills used to assess treatment response for individuals with autism spectrum disorder. Journal of Autism and Developmental Disorders, 43(11), 2491-2501.

Cunningham, A. B. (2012). Measuring change in social interaction skills of young children with autism. Journal of Autism and Developmental Disorders, 42(4), 593-605.

Delemere, E., \& Dounavi, K. (2017). Parent-implemented bedtime fading and positive routines for children with autism spectrum disorders. Journal of Autism and Developmental Disorders. https ://doi.org/10.1007/s10803-017-3398-4.

Estes, A., Munson, J., St. John, T., Dager, S. R., Rodda, A., Botteron, K., ... Guralnick, M. J. (2017). Parent support of preschool peer relationships in younger siblings of children with autism spectrum disorder. Journal of Autism and Developmental Disorders. https ://doi.org/10.1007/s10803-017-3202-5.

Fox, F., Aabe, N., Turner, K., Redwood, S., \& Rai, D. (2017). It was like walking without knowing where I was going: A qualitative study of autism in a UK Somali migrant community. Journal of Autism and Developmental Disorders, 47(2), 305-315. https://doi. org/10.1007/s10803-016-2952-9.

Halstead, E., Ekas, N., Hastings, R. P., \& Griffith, G. M. (2018). Associations between resilience and the well-being of mothers of children with autism spectrum disorder and other developmental disabilities. Journal of Autism and Developmental Disorders. https ://doi.org/10.1007/s10803-017-3447-z.

Larsen, K., Aasland, A., \& Diseth, T. H. (2017). Brief report: Agreement between parents and day-care professionals on early symptoms associated with autism spectrum disorders. Journal of Autism and Developmental Disorders. https://doi.org/10.1007/ s10803-017-3355-2.

Leadbitter, K., Aldred, C., McConachie, H., Le Couteur, A., Kapadia, D., Charman, T., ... Green, J. (2017). The autism family experience questionnaire (AFEQ): An ecologically-valid, parent-nominated measure of family experience, quality of life and prioritised outcomes for early intervention. Journal of Autism and Developmental Disorders. https://doi.org/10.1007/s10803-017-3350-7.

Lindsey, R. A., \& Barry, T. D. (2018). Protective factors against distress for caregivers of a child with autism spectrum disorder. Journal of Autism and Developmental Disorders. https://doi.org/10.1007/ s10803-017-3372-1.

Macari, S. L., Wu, G. C., Powell, K. K., Fontenelle, S., Macris, D. M., \& Chawarska, K. (2017). Do parents and clinicians agree on ratings of autism-related behaviors at 12 months of age? A study of infants at high and low risk for ASD. Journal of Autism and Developmental Disorders. https://doi.org/10.1007/s1080 3-017-3410-z.

Mademtzi, M., Singh, P., Shic, F., \& Koenig, K. (2017). Challenges of females with autism: A parental perspective. Journal of Autism and Developmental Disorders. https://doi.org/10.1007/s1080 3-017-3341-8.

McConachie, H., Livingstone, N., Morris, C., Beresford, B., Le Couteur, A., Gringras, P., ... Parr, J. R. (2017). Parents suggest which indicators of progress and outcomes should be measured in young children with autism spectrum disorder. Journal of Autism and Developmental Disorders. https://doi.org/10.1007/s 1080 3-017-3282-2.

Miller, L. E., Perkins, K. A., Dai, Y. G., \& Fein, D. A. (2017). Comparison of parent report and direct assessment of child skills in toddlers. Research in Autism Spectrum Disorders, 41, 57-65.

Nuttall, A. K., Coberly, B., \& Diesel, S. J. (2018). Childhood caregiving roles, perceptions of benefits, and future caregiving intentions among typically developing adult siblings of individuals with autism spectrum disorder. Journal of Autism and Developmental Disorders. https://doi.org/10.1007/s10803-018-3464-6.

Sacrey, L. A. R., Zwaigenbaum, L., Bryson, S., Brian, J., Smith, I. M., Roberts, W., ... Garon, N. (2018). Parent and clinician agreement regarding early behavioral signs in 12-and 18-month-old infants at-risk of autism spectrum disorder. Autism Research. https://doi. org/10.1002/aur.1920

Shepherd, D., Csako, R., Landon, J., Goedeke, S., \& Ty, K. (2017). Documenting and understanding parent's intervention choices for their child with autism spectrum disorder. Journal of Autism and Developmental Disorders. https://doi.org/10.1007/s1080 3-017-3395-7. 
Shire, S. Y., Shih, W., \& Kasari, C. (2017). Brief report: Caregiver strategy implementation-Advancing spoken communication in children who are minimally verbal. Journal of Autism and Developmental Disorders. https://doi.org/10.1007/s10803-017-3454-0.

Tudor, M. E., Rankin, J., \& Lerner, M. D. (2017). A model of family and child functioning in siblings of youth with autism spectrum disorder. Journal of Autism and Developmental Disorders. https ://doi.org/10.1007/s10803-017-3352-5.

Woodruff, B., Temkit, M. h., Adams, J., \& Yost, K. (2017). Association between affective symptoms and school bullying experiences in adults with autism spectrum disorders (P4. 156). Neurology, 88(16 Supplement), P4. 156. 